Musées, Patrimoine et Culture scientifiques et techniques

159 | 2015

mai-juin 2015

Des politiques européennes aux enjeux locaux : un muséum investi par les chauves-souris

Michèle Lemaire et Laurent Arthur

(2) OpenEdition

Journals

Édition électronique

URL : http://journals.openedition.org/ocim/1513

DOI : $10.4000 /$ ocim. 1513

ISSN : 2108-646X

Éditeur

OCIM

Édition imprimée

Date de publication : 1 mai 2015

Pagination : 16-19

ISSN : 0994-1908

Référence électronique

Michèle Lemaire et Laurent Arthur, « Des politiques européennes aux enjeux locaux : un muséum investi par les chauves-souris », La Lettre de I'OCIM [En ligne], 159 | 2015, mis en ligne le 01 mars 2016, consulté le 02 mai 2019. URL : http://journals.openedition.org/ocim/1513 ; DOI : 10.4000/ocim.1513

Ce document a été généré automatiquement le 2 mai 2019.

Tous droits réservés 


\title{
Des politiques européennes aux enjeux locaux : un muséum investi par les chauves-souris
}

\author{
Michèle Lemaire et Laurent Arthur
}

Espèce anthropophile, le Petit rhinolophe utilise les caves, chaufferies et vide-sanitaires comme site d'hibernation et, à la belle saison, pour établir ses nurseries.

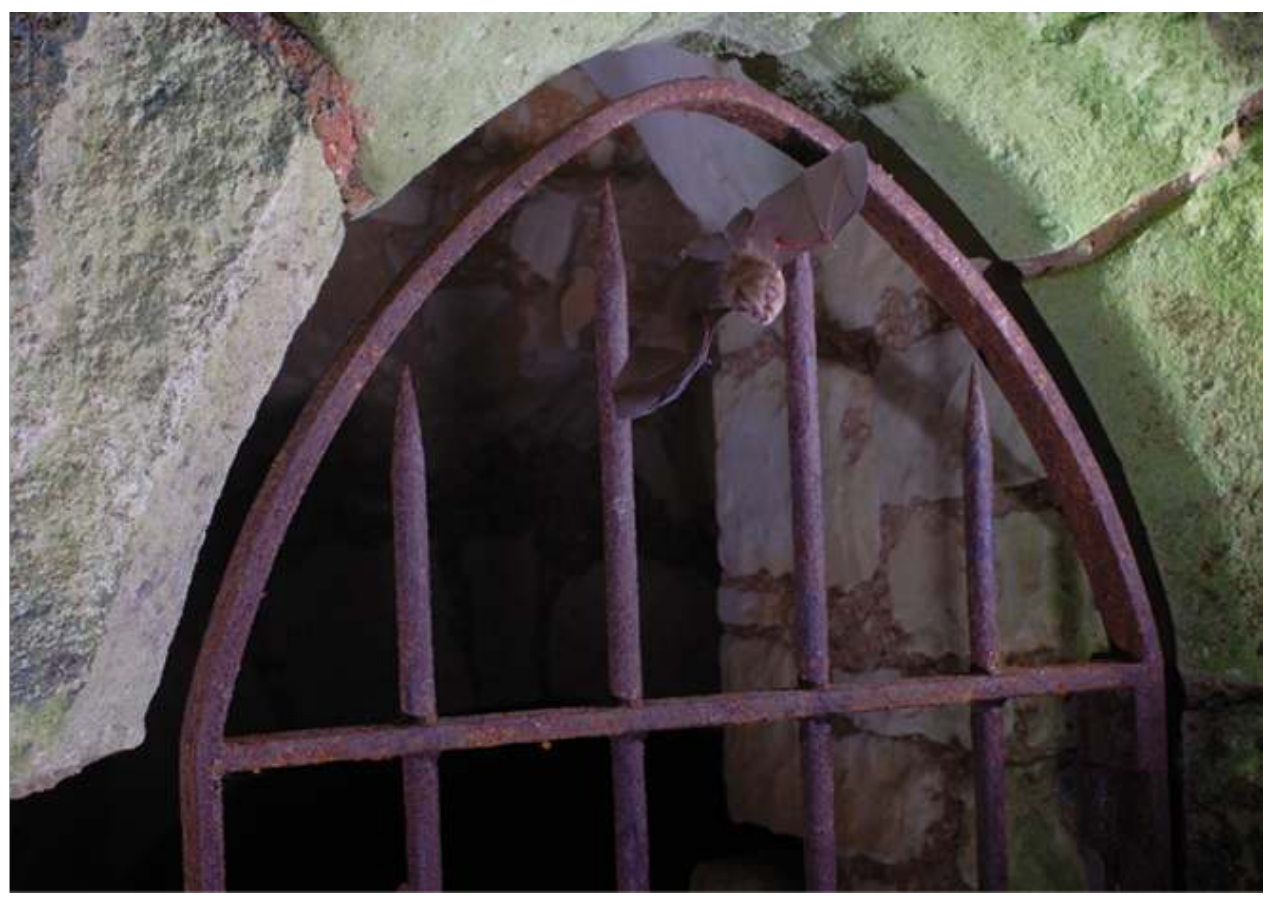

(c) Laurent Arthur

1 Le muséum de Bourges est connu et reconnu depuis de nombreuses années comme un établissement incontournable dans le domaine de la chiroptérologie (Lemaire, 2010). Tout 
spécialiste ou passionné de chauves-souris vient un jour à Bourges pour des rencontres officielles ou de manière indépendante.

Il reçoit chercheurs et étudiants attirés par la connaissance approfondie des colonies des différentes espèces dans leurs milieux. La multiplicité des gîtes de mise-bas connus, plus de 1200 , permet de trouver toutes les variantes nécessaires à quelque projet de recherche que ce soit et ceci, en nombre statistiquement valable. Il y a, par exemple, 143 colonies de Sérotines communes recensées dans le Cher, dans des milieux variés allant de la campagne à la ville. Suivies depuis de nombreuses années, elles permettent d'établir des typologies et des hypothèses quant à leur répartition (Arthur, 2014).

4 Site de médiation, l'intention est de pouvoir répondre aux questionnements de la population. Exposition permanente et site Internet sont prévus pour une première approche. Éditions de livres, participation à divers médias permettent le développement de thématiques. Classes muséum, malles pédagogiques et interventions spontanées lors de la découverte d'un animal dans l'enceinte de l'école visent à toucher les plus jeunes. Sciences participatives (Lemaire, 2012) et réseaux sociaux complètent ce volet médiation ouvert à tous. Mais le plus chronophage restent les interventions ciblées, téléphoniques, via Internet ou en se déplaçant chez l'habitant, pour une meilleure cohabitation entre chauves-souris anthropophiles et propriétaires.

5 Considérés comme experts, les deux spécialistes du muséum sont intégrés également dans différentes instances comme le secrétariat Eurobats, chargé de l'accord sur la conservation des chauves-souris en Europe, le comité de pilotage du Plan national d'action pour les chauves-souris (PNA) ou auprès de l'UICN (Union internationale pour la conservation de la nature) pour l'établissement des listes rouges nationales.

6 Le muséum intervient de ce fait à tous les niveaux, de la gouvernance européenne ou nationale jusqu'aux problématiques locales.

\section{Au service du réseau national des chiroptérologues, le muséum apporte du lien}

7 Tous les deux ans sont organisées à Bourges les Rencontres nationales chauve-souris. En alternance avec les Rencontres des grandes régions (Grand-Ouest, Grand-Sud et GrandEst) préparées par les groupes régionaux. Événement unificateur depuis 25 ans, les participants des Rencontres nationales viennent toujours plus nombreux et la dernière manifestation de 2014 a fait converger 450 passionnés en provenance des pays francophones. Ouvertes à tous, ces Rencontres sont construites sous forme d'une journée technique prise en charge par la Société Française pour l'Étude et la Protection des Mammifères (SFEPM) et de deux jours de séances plénières et d'ateliers dont le but est de répondre, grâce à des thématiques hétérogènes, aux questions des débutants comme aux besoins d'échanges des professionnels ou des amateurs éclairés.

8 Organe de centralisation, la bibliothèque du muséum est chargée de rassembler la documentation nationale, $\mathrm{du}$ plus petit article paru dans un bulletin local aux publications scientifiques référencées. Le muséum joue son rôle de centre d'information et de mémoire en éditant chaque année une liste bibliographique la plus exhaustive 
possible, présentant les articles répertoriés depuis Pierre Belon en 1555 jusqu'à nos jours, et en réceptionnant les articles fournis par le réseau pour les mettre à disposition de tous (www.museum-bourges.net/chauve-souris-centre-de-documentation-80.html). C'est une action du PNA, validée autant par le ministère de l'Écologie, du Développement durable et de l'Énergie que par le réseau national, et elle est partagée avec le muséum d'Histoire naturelle de Genève qui a la charge de la bibliographie scientifique mondiale.

La Pipistrelle commune est l'une des deux espèces qui nécessite le plus de médiation pour une cohabitation harmonieuse. Ici, une colonie de femelles avec leurs petits dans une toiture de maison.

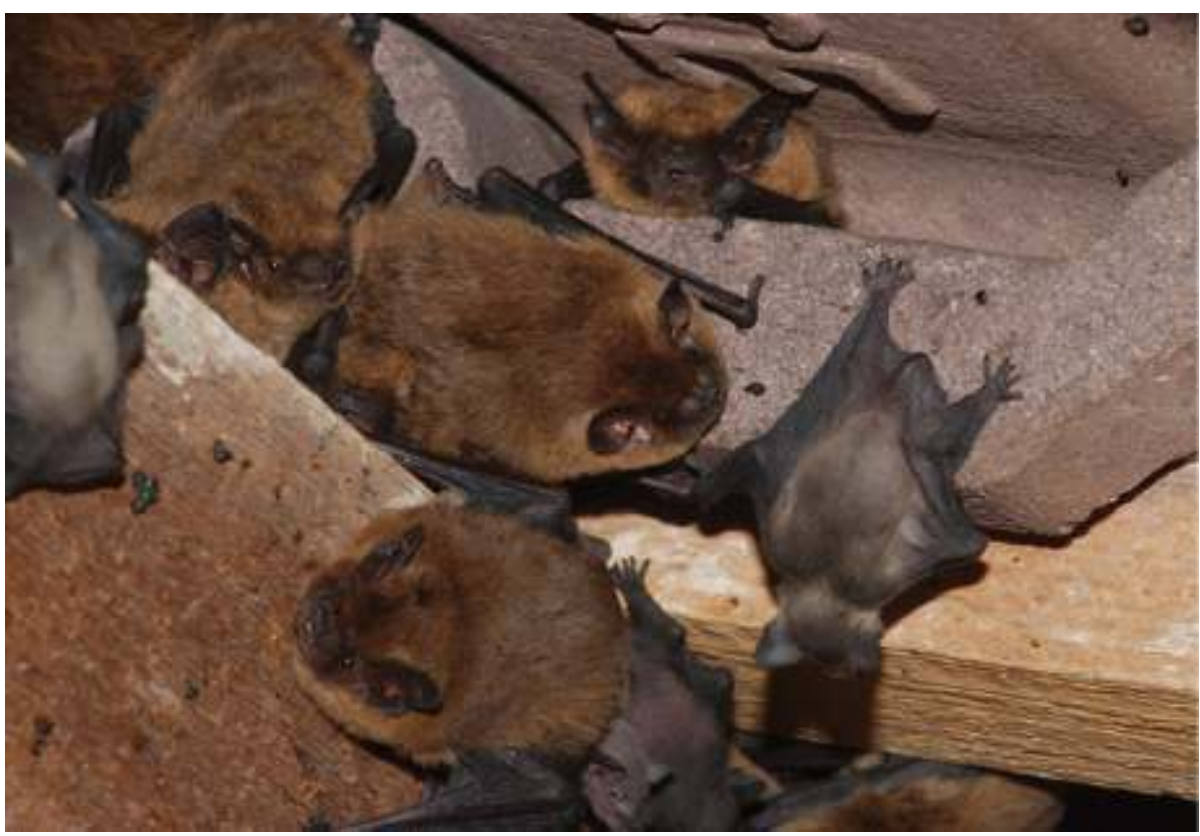

(c) Laurent Arthur

\section{Au service des politiques}

Rattaché au maire-adjoint chargé de l'écologie, le muséum est considéré par la collectivité comme un acteur concret de la biodiversité. À la croisée de la vie culturelle et du monde environnemental, le muséum apporte ses compétences dans le cadre des politiques environnementales et diffuse les connaissances au sein de la vie culturelle de la région. Trois exemples seront présentés ici.

Par délégation de l'État, les Collectivités ont la charge de soutenir et mettre en œuvre certaines actions environnementales. Les sites Natura 2000 font partie de ces contrats que les municipalités peuvent gérer.

La Ville de Bourges est ainsi maitre d'ouvrage pour le site "Natura 2000 - FR2400516 Carrières de Bourges ", bien que les cavités concernées ne soient pas toutes sur l'emprise de la commune (http://inpn.mnhn.fr/site/natura2000/FR2400516). La Ville, représentée par son maire-adjoint chargé de l'écologie, assure la présidence du comité de pilotage alors que le muséum, service municipal, gère le secrétariat et le suivi du déroulement de ce contrat. Cependant l'animation est sous-traitée à un autre organisme car le personnel du muséum ne peut le prendre en charge, faute de disponibilité. Et la question se pose pour certains responsables d'établissement quant au rôle d'un muséum de prendre en 
charge ce type d'animation (rencontre de propriétaires, rédaction de charte, négociation de travaux favorables à la biodiversité).

\section{Des Trames vertes et bleues aux Trames noires}

12 La notion de Trames noires est née à Bourges quand le Grenelle de l'Environnement a oublié cette couleur dans le concept des Trames vertes et blenes (voir encadré). Pour toutes les espèces lucifuges, cet oubli de taille pouvait avoir des conséquences néfastes sur le long terme face à l'étalement urbain croissant et au développement des éclairages publics dans un but sécuritaire, et ce jusqu'au plus profond des hameaux. Un corridor écologique de qualité coupé par des zones éclairées perdrait alors tout intérêt pour un tiers des mammifères nationaux, sans compter les autres embranchements touchés, oiseaux, amphibiens ou invertébrés nocturnes. De l'échelle régionale, avec les schémas régionaux de cohérence écologique (SRCE), en passant par les schémas de cohérence territoriale (SCOT), jusqu'au niveau du plan local d\&apos ;urbanisme (PLU) d'une ville, ces Trames noires sont superposées aux vertes et bleues par les politiques ayant compris l'intérêt synergique sous-jacent pour la biodiversité, notamment en milieu urbain.

Depuis, les études des futurs tracés routiers tendent à intégrer des zones d'ombres pour permettre les déplacements fluides des chauves-souris entre leurs divers territoires. Cependant, des études à l'application sur le terrain, il reste parfois encore des efforts à développer.

Les questionnements sur la lumière et les chiroptères (seuil de tolérance ou incidence de la luminosité artificielle sur les gîtes et les territoires de chasse) sont venus naturellement et de manière additionnelle se greffer aux Trames noires, une nouvelle spécialité où le muséum de Bourges s'investit vis-à-vis de ces espèces. 
Entre traitements phytosanitaires et faune sauvage, il faudra trouver un juste équilibre.

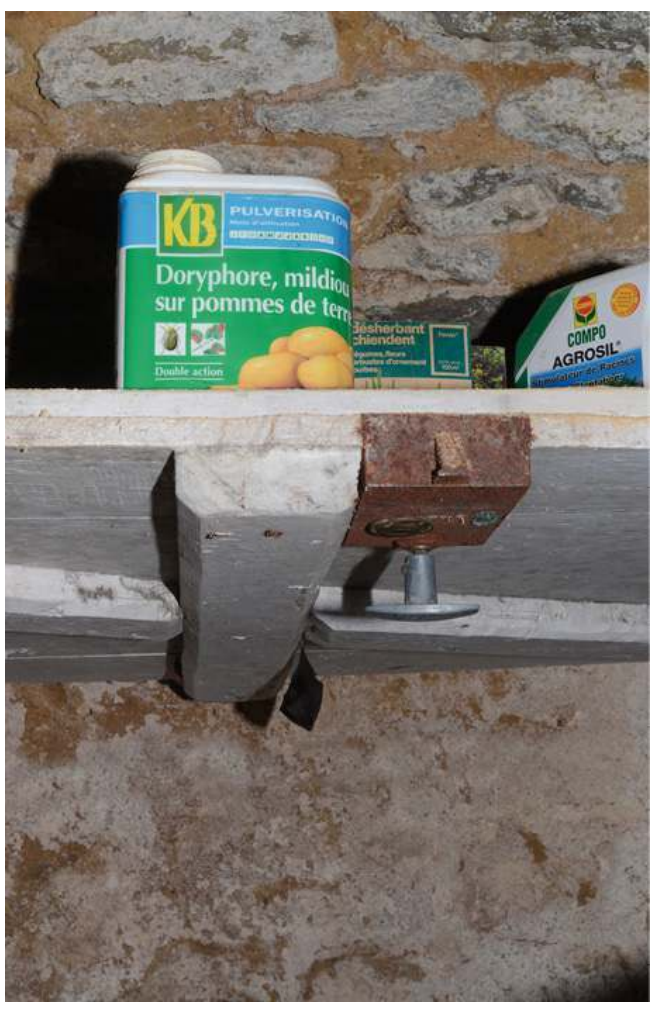

(c) Laurent Arthur

\section{Les ponts du Conseil départemental}

15 Les ouvrages d'art gérés par le Conseil départemental du Cher et soumis à travaux sont suivis depuis plus de 20 ans par les spécialistes du muséum. Les chauves-souris présentes dans ces structures sont recensées et les ponts à enjeux sont intégrés par les services des routes dans une base informatisée. Tout ouvrage soumis à travaux est signalé au muséum qui intervient dès les avant-projets puis suit le chantier jusqu'à son exécution finale en conservant sur place des gîtes accessibles pour les animaux. Une trentaine de ponts ont ainsi été rénovés et permettent la sauvegarde d'autant de colonies et de plus d'un millier d'individus de huit espèces adeptes de la vie sous les ponts.

16 Afin d'aller encore plus loin, les ouvrages neufs sont aujourd'hui équipés systématiquement de gîtes dans la maçonnerie sur les secteurs à forts enjeux environnementaux. Des séances de formations pour les services techniques du Conseil départemental du Cher sont organisées par le muséum pour faire connaître les dernières avancées techniques et les résultats qui motiveront d'autant plus les donneurs d'ordre et les gestionnaires des routes. 
Noctule de Leisler dans sa cavité arboricole. Il arrive qu'elle pénètre dans les habitations aux intersaisons, notamment dans les inserts et les poêles à bois, piégée par les tubages lisses.

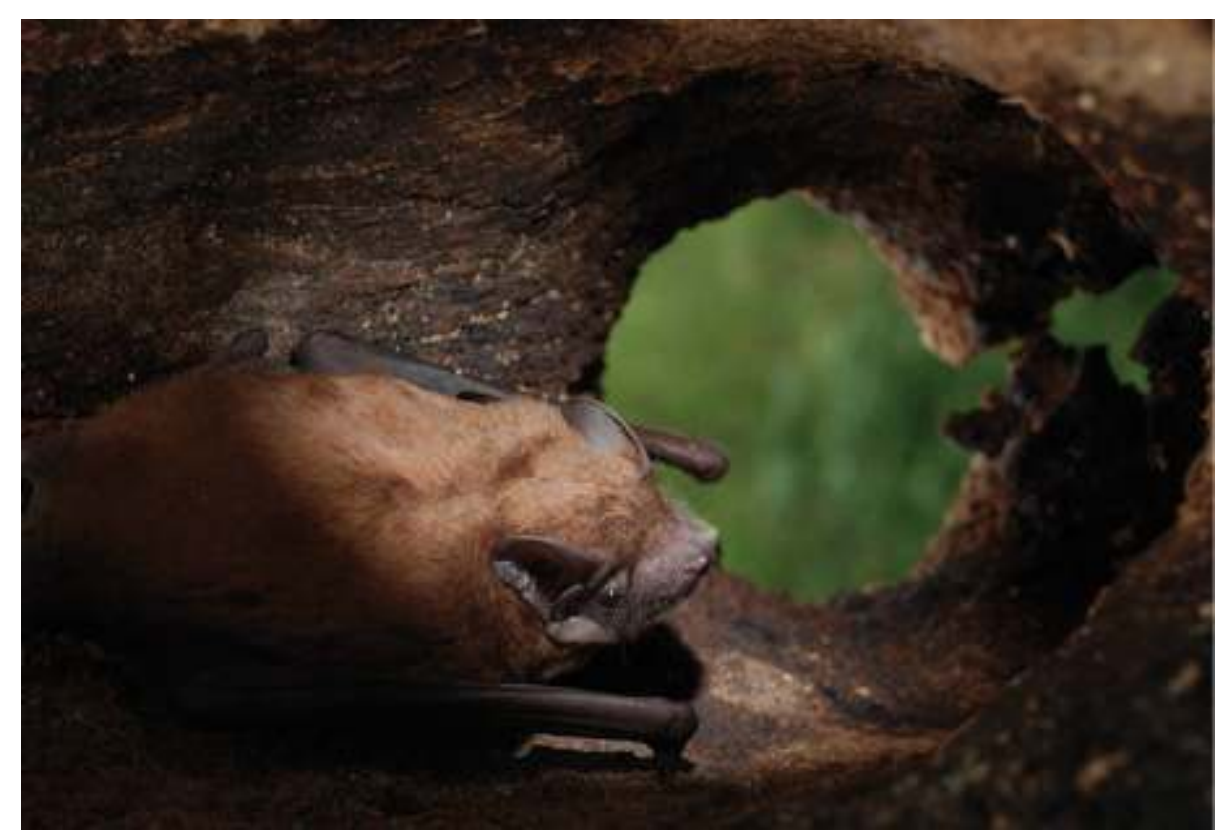

(C) Laurent Arthur

\section{Au service de la population}

17 Autre volet, les interventions auprès des personnes ayant des problèmes de cohabitation avec des espèces sauvages installées dans les bâtiments. Au-delà d'une médiation classique qui consiste à faire comprendre la faune sauvage, rassurer, apprécier et donner des arguments objectifs de cohabitation, certains cas nécessitent des aménagements concrets pour qu'un partage d'un même espace entre humain et chauves-souris soit envisageable sur le long terme (Arthur, 2015). Il s'agit de traiter annuellement 1500 demandes, tous appels et interventions confondus, sur l'ensemble du département, sans compter les sollicitations nationales.

\section{Conclusion}

Si le muséum de Bourges est reconnu dans le domaine des chauves-souris, l'ampleur de cette spécialité n'était pas initialement prévue, elle s'est construite au fil du temps et d'événements, souvent fortuits. Avoir cette image a permis de pénétrer les médias et d'avancer plus facilement dans une politique de communication donnant une identité au muséum auprès des publics et des instances nationales (Minchin, 1996). Mais cette image de marque n'est pas sans inconvénient et ne doit pas occulter les nombreuses autres actions développées au sein de la structure. La responsabilité de la direction de l'établissement est de trouver un équilibre entre notoriété à effet positif et maintien de la diversité des rôles d'un muséum d'Histoire naturelle.

Une des raisons qui a contribué à un tel essor de cette activité est le besoin du citoyen qui ne sait pas où s'adresser lors de questionnements ou de problèmes engendrés par le 
sauvage. De même, les collectivités recherchent des interlocuteurs face à des problématiques auxquelles ils ont aujourd'hui des obligations légales de résultats. Répondre à ces multiples sollicitations dépasse largement le périmètre sur lequel le muséum est censé travailler pour sa collectivité, et la tolérance du politique se positionne dans un équilibre entre effet positif pour la collectivité et investissement raisonnable en temps et financements.

$\mathrm{Au}$ final, des directeurs de muséums s'interrogent: jusqu'où ces implications environnementales et ce rôle d'expert ou de médiateur sont-ils un terrain d'activités pour une structure comme un muséum?

\section{La Trame verte et bleue}

La Trame verte et bleue est une mesure phare du Grenelle Environnement qui porte l'ambition d'enrayer le déclin de la biodiversité au travers de la préservation et de la restauration des continuités écologiques.

La Trame verte et bleue est un outil d'aménagement du territoire qui vise à (re)constituer un réseau écologique cohérent, à l'échelle du territoire national, pour permettre aux espèces animales et végétales, de circuler, de s'alimenter, de se reproduire, de se reposer... En d'autres termes, d'assurer leur survie, et permettre aux écosystèmes de continuer à rendre à l'homme leurs services. Les continuités écologiques correspondent à l'ensemble des zones vitales (réservoirs de biodiversité) et des éléments (corridors écologiques) qui permettent à une population d'espèces de circuler et d'accéder aux zones vitales. La Trame verte et bleue est ainsi constituée des réservoirs de biodiversité et des corridors qui les relient.

(source: Ministère de l'Écologie, du Développement durable et de l'Énergie, www.developpementdurable.gouv.fr)

\section{La Trame verte et bleue}

\section{BIBLIOGRAPHIE}

Arthur, L., Lemaire, M., Dufrêne, L., Le Viol, I., Julien, J.-F. et Kerbiriou, C. Understanding bathabitat associations and the effects of monitoring on long-term roost success using a volunteer dataset, Acta chiropterologica, vol. 16, nº 2, 2014, pp. 397-412.

Arthur, L. et Lemaire, M. Les chauves-souris de France, Belgique, Luxembourg et Suisse. Biotope, MNHN, 2015, 544 p.

Lemaire, M. Le muséum de Bourges un référent naturel pour les chauves-souris de France, Musées et collections publiques de France, $\mathrm{n}^{\circ} 258,2010$, pp. 54-59.

Lemaire, M. Le muséum d'Historie naturelle de Bourges et la biodiversité urbaine, La Lettre de l'OCIM, n' 144, 2012,

pp. 52-59. 
Minchin, S. Les muséums d'Histoire naturelle ont-ils intérêt à se doter d'une image ?Étude du cas muséum d'Histoire naturelle de Bourges-chauves-souris. DEA Muséologie des sciences de la nature et de l'homme, MNHN, 1996, 79 p.

\section{RÉSUMÉS}

Référence pour l'étude des chauves-souris et l'ensemble de la documentation sur le sujet, le muséum d'Histoire naturelle de Bourges est également un médiateur incontournable auprès des collectivités territoriales et des citoyens pour la bonne gestion de ces diverses populations d'animaux nocturnes: les auteurs s'interrogent sur l'harmonisation de ces activités avec les autres missions dévolues habituellement à un muséum.

INDEX

Mots-clés : muséum, chauve-souris

\section{AUTEURS}

\section{MICHĖLE LEMAIRE}

conservatrice du muséum d'Histoire naturelle de Bourges michele.lemaire@ville-bourges.fr

\section{LAURENT ARTHUR}

conservateur au muséum d'Histoire naturelle de Bourges

laurent.arthur@ville-bourges.fr 